\title{
Population-Based Study on Cancer Subtypes, Guideline-Concordant Adjuvant Therapy, and Survival Among Women With Stage I-III Breast Cancer
}

\author{
Mei-Chin Hsieh, PhDa; Lu Zhang, PhDa; Xiao-Cheng Wu, MD, MPHª; Mary B. Davidson, MNa; \\ Michelle Loch, MD' ${ }^{b}$ and Vivien W. Chen, PhDa
}

\section{ABSTRACT}

Background: Breast cancer subtype is a key determinant in treat-
ment decision-making, and also effects survival outcome. In this
population-based study, in-depth analyses were performed to ex-
amine the impact that breast cancer subtype and receipt of guideline-
concordant adjuvant systemic therapy (AST) have on survival using a
population-based cancer registry's data. Methods: Women aged
$\geq 20$ years with microscopically confirmed stage I-III breast cancer
diagnosed in 2011 were identified from the Louisiana Tumor Registry.
Breast cancer subtypes were categorized based on hormone re-
ceptor (HR) and HER2 status. Guideline-concordant treatment was
defined using the NCCN Guidelines for Breast Cancer. Logistic
regression was applied to identify factors associated with guideline-
concordant AST receipt. Kaplan-Meier survival curves were gener-
ated to compare survival among subtypes by AST receipt status, and
a semiparametric additive hazard model was used to verify the factors
impacting survival outcome. Results: Of 2,214 eligible patients, most
(70.8\%) were HR+/HER2- followed by HR-/HER2- (14.4\%), and
$78.6 \%$ received guideline-concordant AST. Compared with patients
with the HR+/HER2+ subtype, women with other subtypes were
more likely to be guideline-concordant after adjusting for socio-
demographic and clinical variables. Women with the HR-/HER2+
or HR-/HER2- subtype had a higher risk of any-cause and breast
cancer-specific death than those with the HR+/HER2+ subtype.
Those who did not receive AST had an additional adjusted hazard
of 0.0191 ( $P=.0001$ ) in overall survival and 0.0126 ( $P=.0011$ ) in
cause-specific survival compared with those who received AST.
Conclusions: Most patients received guideline-concordant AST,
except for those with the HR+/HER2+ subtype. Patients receiving
guideline-adherent adjuvant therapy had better survival outcomes
across all breast cancer subtypes. J Natl Compr Canc Netw 2019;17(6):676-686 doi: $10.6004 /$ jnccn.2018.7272

\footnotetext{
aEpidemiology Program and Louisiana Tumor Registry, School of Public Health, and ${ }^{\mathrm{b} H e m a t o l o g y}$ and Oncology, School of Medicine, Louisiana State University Health Sciences Center, New Orleans, Louisiana.
}

\section{Background}

Since the molecular phenotypes of breast carcinomas were distinguished by gene expression patterns in 2000 , breast cancer is no longer considered a single disease but rather a group of molecularly distinct neoplastic disorders. ${ }^{1-4}$ Based on hormone receptor (HR) status, including estrogen receptor (ER) and progesterone receptor (PR), HER2 status, and proliferation markers or histologic grade, 4 distinct intrinsic molecular subtypes of breast cancer have been identified: luminal A, luminal B, basal-like (triple-negative: ER-/PR-/HER2-), and HER2+/ER-. ${ }^{1,4}$ These subtypes vary by race and influence survival rate. ${ }^{5}$

When physicians prescribe appropriate adjuvant systemic therapies (ASTs) for patients with breast cancer, molecular subtypes are a key determinant. Clinical trials have demonstrated that patients with $\mathrm{HR}+(\mathrm{ER}+$ and/or $\mathrm{PR}+$ ) breast cancer benefit from tamoxifen, those with HR- (ER- and PR-) benefit from adjuvant chemotherapy, and those with HER2 + disease benefit from trastuzumab by decreasing recurrence and increasing survival. ${ }^{6-9}$ The significance of these tumor markers led ASCO in 2007 to recommend measuring ER/PR and HER2 expression and/or amplification in every primary invasive breast cancer. ${ }^{10}$ In addition, the NCCN Clinical Practice Guidelines in Oncology (NCCN Guidelines) for Breast Cancer have taken ER/PR and HER2 status into account in guiding clinical care. ${ }^{11}$

Few population-based studies have examined the association of breast cancer subtypes with guidelineconcordant AST and survival. Studies on guidelineconcordant AST have mainly focused on adjuvant hormone therapy and chemotherapy, which was based on HR (ER and PR) status, ${ }^{12,13}$ or examined the use of trastuzumab only. ${ }^{14}$ No studies have examined AST with hormone therapy, chemotherapy, and trastuzumab together by breast cancer subtype. The goal of the present study was to investigate whether receipt of guideline-concordant AST varied by breast cancer 
subtype after adjusting for patients' sociodemographic and tumor characteristics, and to examine the impact of guideline-concordant treatment status and cancer subtype on survival outcome.

\section{Methods}

\section{Data Source and Study Cohort}

Data on female breast cancer were obtained from the Louisiana Tumor Registry (LTR), a population-based specialized cancer registry funded by the Centers for Disease Control and Prevention's (CDC's) National Program of Cancer Registries that participates in the CDC-funded project Enhancing Cancer Registry Data for Comparative Effectiveness Research (CER) and the SEER registry. The CDC-CER registries reabstracted medical records and collected detailed and complete first-course treatment information, including type of surgery, radiation, and all systemic treatments, from all sources for patients diagnosed with breast cancer in $2011 .{ }^{15}$ Because gene expression profiling is not performed routinely in many healthcare facilities or collected by populationbased cancer registries, ER, PR, and HER2 status based on immunohistochemistry and/or fluorescence in situ hybridization (FISH) results are commonly used as surrogates to categorize breast cancer into 4 subtypes: $\mathrm{HR}+\mathrm{I}$ HER2+, HR+/HER2-, HR-/HER2+, and HR-/HER2-. ${ }^{16}$

Eligible patients were women with breast cancer aged $\geq 20$ years with microscopically confirmed stage I-III disease diagnosed in 2011 and who had undergone total mastectomy or breast-conserving surgery plus radiation. Eligibility was restricted to patients with histologic codes 8050, 8140, 8201, 8211, 8401, 8480, 8481, and $8500-8575$ and with breast cancer as the only, first, or second primary cancer. Women who died within 30 days after surgery were excluded. A total of 32 patients were diagnosed with either bilateral breast cancers or 2 ipsilateral primary tumors in 2011; only the first primary was included. Selection of eligible patients is detailed in Figure 1.

\section{Definition of Guideline-Concordant AST}

Systemic therapies included in this study were hormonal, chemotherapy, and trastuzumab. Receipt of guidelineconcordant AST was defined as treatment based on the NCCN Guidelines for Breast Cancer, ${ }^{11}$ which takes into account cancer stage, histology, and subtype; tumor size; lymph node status; and risk score of recurrence (Table 1) for pathologically staged breast cancer. Patients who underwent total mastectomy or breast-conserving surgery plus radiation and received NCCN-recommended systemic therapy were categorized as guideline-concordant.

The multigene signature results or 21-gene recurrence score was used to determine whether chemotherapy was required for patients with $\mathrm{HR}+$,

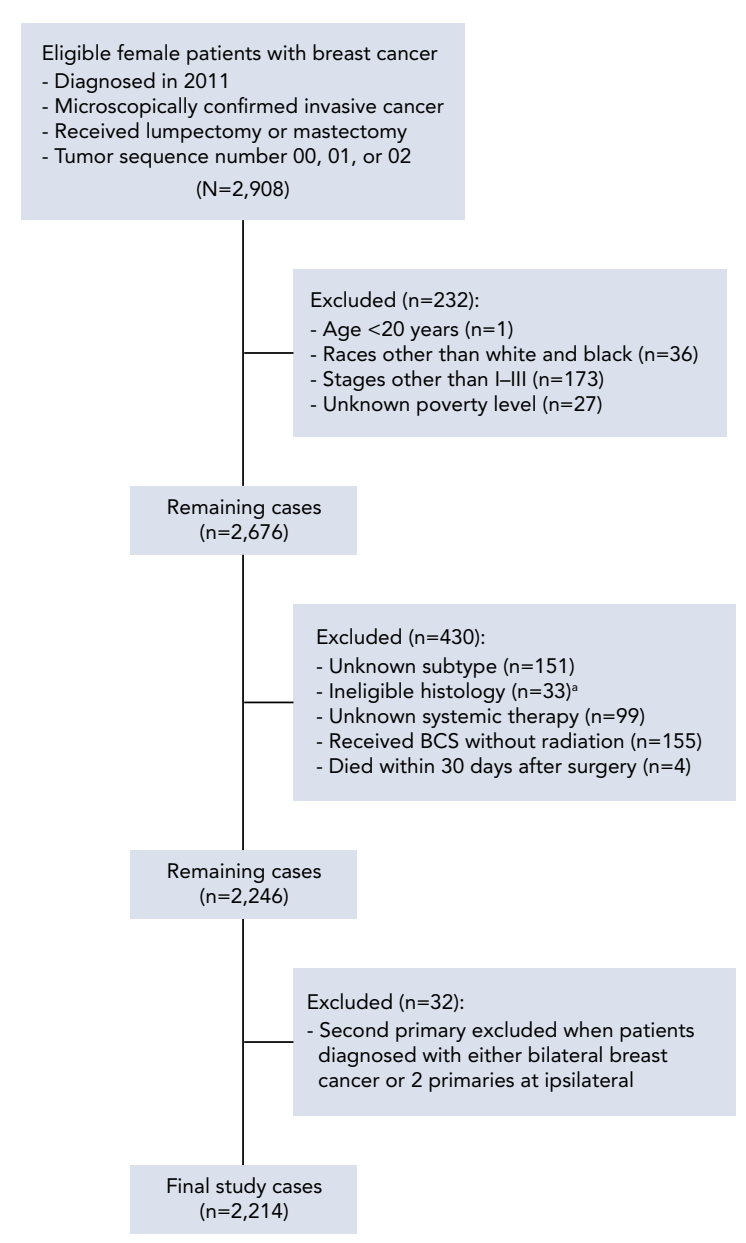

Figure 1. Flow chart of eligible study cohort selection. aHistologies other than those with codes 8050, 8140, 8201, 8211, 8401, 8480, 8481 , and $8500-8575$ were ineligible.

Abbreviation: BCS, breast-conserving surgery.

node-negative, early-stage breast cancer. ${ }^{11,17,18}$ Patients with early-stage HR+/HER2- breast cancer with tumor size $>0.5 \mathrm{~cm}$ and negative lymph nodes were required to have chemotherapy if the recurrence score was $\geq 31$ or there was a high risk of recurrence. In addition to routinely collected recurrence scores for breast cancer cases, LTR linked its breast cancer cases with the Oncotype DX (Genomic Health) database to obtain the recurrence risk score that was not captured in the routine data collection. In patients with node-negative, HR+/HER2- disease and tumor size $>0.5 \mathrm{~cm}$ for whom the recurrence score assay was not performed, the NCCN Guidelines recommend chemotherapy be considered. ${ }^{11}$ For patients aged $>70$ years with a subtype and tumor condition in whom adjuvant chemotherapy was an option, the guidelines suggest that chemotherapy be individualized based on the patient's comorbid conditions. ${ }^{11}$ Hence, we used Deyo's enhanced Charlson comorbidity score (CCS) ${ }^{19}$ to determine whether chemotherapy should be 


\section{Table 1. Recommended AST for Stage I-III Breast Cancer With Mastectomy or BCS Plus Radiation}

\begin{tabular}{|c|c|c|c|c|c|}
\hline Subtype & Lymph Node & Tumor Size & Hormone & Chemotherapy & Trastuzumab \\
\hline \multicolumn{6}{|c|}{ Stage I, IIA, IIB, IIIA with N1; histology not tubular and colloid } \\
\hline \multirow{4}{*}{$\mathrm{HR}+/ \mathrm{HER} 2+$} & \multirow{3}{*}{ pNo; pN1mi ( $\leq 2 \mathrm{~mm})$} & $\leq 0.5 \mathrm{~cm}$ or $\mathrm{Ml}$ & pNO: Yes-c; pN1mi: Yes & pN1mi: Yes-c & pN1mi: Yes \\
\hline & & $0.6-1.0 \mathrm{~cm}$ & Yes & Yes-c & Yes \\
\hline & & $>1.0 \mathrm{~cm}$ & Yes & Yes & Yes \\
\hline & Node-positive & & Yes & Yes & Yes \\
\hline \multirow[t]{4}{*}{$\mathrm{HR}+/ \mathrm{HER} 2-$} & pNO & $\leq 0.5 \mathrm{~cm}$ or $\mathrm{Ml}$ & Yes-c & & \\
\hline & $\mathrm{pN} 1 \mathrm{mi}(\leq 2 \mathrm{~mm})$ & $\leq 0.5 \mathrm{~cm}$ or $\mathrm{Ml}$ & Yes & & \\
\hline & pNo; pN1mi ( $\leq 2 \mathrm{~mm})$ & $>0.5 \mathrm{~cm}$ & Yes & Yes $^{\mathrm{a}}$ & \\
\hline & Node-positive & & Yes & Yes & \\
\hline \multirow[t]{5}{*}{ HR-/HER2+ } & pNO & $\leq 0.5 \mathrm{~cm}$ or $\mathrm{Ml}$ & & & \\
\hline & $\mathrm{pN} 1 \mathrm{mi}(\leq 2 \mathrm{~mm})$ & $\leq 0.5 \mathrm{~cm}$ or $\mathrm{Ml}$ & & Yes-c & Yes-c \\
\hline & \multirow[t]{2}{*}{ pNo; pN1mi ( $\leq 2 \mathrm{~mm})$} & $0.6-1.0 \mathrm{~cm}$ & & Yes-c & Yes-c \\
\hline & & $>1.0 \mathrm{~cm}$ & & Yes & Yes \\
\hline & Node-positive & & & Yes & Yes \\
\hline \multirow[t]{5}{*}{ HR-/HER2- } & pNO & $\leq 0.5 \mathrm{~cm}$ or $\mathrm{Ml}$ & & & \\
\hline & $\mathrm{pN} 1 \mathrm{mi}(\leq 2 \mathrm{~mm})$ & $\leq 0.5 \mathrm{~cm}$ or $\mathrm{Ml}$ & & Yes-c & \\
\hline & $\mathrm{pNO}$; pN1mi ( $\leq 2 \mathrm{~mm})$ & $0.6-1.0 \mathrm{~cm}$ & & Yes-c & \\
\hline & & $>1.0 \mathrm{~cm}$ & & Yes & \\
\hline & Node-positive & & & Yes & \\
\hline \multicolumn{6}{|c|}{ Stage I, IIA, IIB, IIIA with N1; histology tubular or colloid } \\
\hline \multirow[t]{4}{*}{$\mathrm{HR}+$} & \multirow[t]{3}{*}{ pNo; pN1mi ( $\leq 2 \mathrm{~mm})$} & $<1.0 \mathrm{~cm}$ & & & \\
\hline & & $1.0-2.9 \mathrm{~cm}$ & Yes-c & & \\
\hline & & $\geq 3.0 \mathrm{~cm}$ & Yes & & \\
\hline & Node-positive & & Yes & Yes-c & \\
\hline HR- & $\begin{array}{l}\text { Treat as HR- for histology } \\
\text { not tubular and colloid }\end{array}$ & & & & \\
\hline \multicolumn{6}{|c|}{ Stage IIIA with N2+, IIIB, IIIC } \\
\hline $\mathrm{HR}+/ \mathrm{HER} 2+$ & NA & NA & Yes & Yes & Yes \\
\hline HR+/HER2- & NA & NA & Yes & Yes & \\
\hline HR-/HER2+ & NA & NA & & Yes & Yes \\
\hline HR-/HER2- & NA & NA & & Yes & \\
\hline
\end{tabular}

Data per the NCCN Guidelines for Breast Cancer. ${ }^{11}$

Abbreviations: AST, adjuvant systemic therapy; BCS, breast-conserving surgery; HR, hormone receptor; MI, microinvasive; NA, not applicable; Yes-c, considered adjuvant.

${ }^{a}$ Chemotherapy is considered if the 21-gene recurrence score is $18-30$. Chemotherapy is required if the recurrence score is $\geq 31$.

recommended for these patients. If the patients had any type of Charlson comorbidity, we assumed that adjuvant chemotherapy was not recommended.

\section{Survival}

This study assessed overall and breast cancer-specific survival. Eligible patients were followed up to December 31,2017 , if alive. Survival duration was the time between date of initial diagnosis until date of death, date of last contact, or closing follow-up if alive. The underlying cause of death for deceased patients was obtained from the state or the national death file. The event for overall survival was death from any cause. The definition of cause-specific survival for cases with tumor sequences 00 and 01 was based on the SEER cause-specific death classification using ICD- $10,{ }^{20}$ whereas for sequence 02 cases, only patients with breast cancer as a cause of death were selected. Patients who died of other causes were censored. 


\section{Covariates}

Patient demographic variables included race (white, black), age at diagnosis ( $<50,50-59,60-69,70-79, \geq 80$ years), insurance status at diagnosis, marital status, and neighborhood poverty level $(<10 \%, 10 \%-19.9 \%, \geq 20 \%)$ at the census tract address at diagnosis. Clinical variables included AJCC stage (I, II, III) using the 7th edition of the AJCC Cancer Staging Manual, tumor sequence (00: 1 primary; 01 or $02:>1$ primary), Bloom-Richardson grade (low, intermediate, high, unknown grade), and Deyo's enhanced CCS (no comorbidity documented, no Charlson comorbidity, CCS 1, CCS $\geq 2$ ). Deyo's enhanced CCS was calculated based on comorbid conditions coded in medical records using ICD-9-CM diagnosis codes. Coding instructions and valid ICD-9-CM diagnosis codes for preexisting comorbidity were based on the Facility Oncology Registry Data Standards (FORDS) manual. ${ }^{21}$ The category "comorbidity not documented" included patients without a comorbid condition found in medical records or with an ICD-9-CM diagnosis code not included in the FORDS list.

\section{Statistical Analysis}

Descriptive statistics on breast cancer subtype, patient sociodemographic factors, and tumor characteristics by receipt of guideline-concordant AST were assembled, and the Pearson's chi-square test was used to assess the association. We used logistic regression to examine the association between cancer subtype and receipt of guideline-concordant AST for both unadjusted and adjusted models. The Kaplan-Meier method was used to estimate survival time by breast cancer subtype and AST receipt status, and statistically significant difference was based on the log-rank test. Although the Cox proportional hazards regression model, which estimates relative change, is the most popular model to estimate the hazard of an event occurring, it is a challenge to meet the proportional hazard assumption for a model that involves many covariates and assesses 2 different survival outcomes. Instead, we used the semiparametric additive hazard regression (SAHR) model ${ }^{22,23}$ to estimate the regression parameter; this model can estimate the absolute change in risk rather than the relative change. Cox-Snell residuals were used to evaluate the overall fit of the SAHR model. If the model fits the data well, then the Cox-Snell residuals should follow a unit exponential distribution. ${ }^{24,25}$ All analyses were performed using SAS 9.4 (SAS Institute Inc.), and statistical tests of significance were based on a 2-sided test with significance levels of 0.05 .

\section{Results}

A total of 2,214 eligible female patients with breast cancer were included in the study: 1,582 $(71.5 \%)$ were white and $632(28.5 \%)$ were black, and $496(22.4 \%)$ of them had either tumor sequence number 01 (192 cases) or 02 (304 cases) (Table 2). Most patients were HR+/HER2(70.8\%), followed by HR-/HER2- (14.4\%), HR +/HER2+ $(10.0 \%)$, and HR-/HER2+ $(4.8 \%)$. The mean age was 60.0 years for HR+/HER2 +, 61.9 years for HR + /HER2-, 56.6 years for HR-/HER2+, and 57.1 years for HR-/HER2-. Of 1,568 patients with HR+/HER2-, 35.6\% had a recurrence score available and $3.4 \%$ were at high risk of recurrence.

\section{Guideline-Concordant AST}

An average of $78.6 \%$ of the study cohort received guidelineconcordant AST. Women with the HR+/HER2+ subtype had the lowest percentage of receipt of adjuvant chemotherapy, hormone treatment, and trastuzumab (Figure 2) among the subtypes and $>50 \%$ of patients with the HR+ I HER2- subtype either were considered for chemotherapy or did not require chemotherapy. Compared with patients with the HR+/HER2+ subtype, patients with other subtypes were more likely to receive guideline-concordant AST with an adjusted odds ratio (aOR) of 4.08 (95\% CI, 2.94-5.67) for HR+/HER2-, 3.68 (95\% CI, 2.06-6.57) for HR-/HER2+, and 3.46 (95\% CI, 2.29-5.23) for HR-/HER2after controlling for other factors (Table 2). Among patients sociodemographic factors, only age at diagnosis and poverty level were significantly associated with receipt of guideline-concordant AST (Table 2). Compared with patients aged $<50$ years, those aged $70-79$ and $\geq 80$ years had a $49 \%$ (aOR, 0.51; 95\% CI, 0.34-0.75) and 78\% (aOR, 0.22; 95\% CI, 0.14-0.35) lower likelihood of receiving AST after adjusting for other covariants. Patients residing in a census tract $\geq 20 \%$ below poverty were more likely to be guideline-concordant (aOR, 1.47; 95\% CI, 1.09-1.98) than those residing in a census tract $<10 \%$ below poverty.

The percentage of patients receiving AST decreased in more advanced stages of disease. Women with stage IIA disease (T0N1M0/T1N1M0/T2N0M0) had a lower likelihood of receiving AST than those with stage IA (T1N0M0), with aOR 0.51 (95\% CI, 0.38-0.67), whereas in women with stage IIIB disease (tumor directly extends to chest wall and/or skin), the aOR decreased to 0.11 (95\% CI, 0.05-0.22). We also observed that women with a CCS of 1 were more likely to receive AST than those without a CCS (aOR, 1.82; 95\% CI, 1.28-2.58), whereas other comorbid groups showed no statistically significant difference.

\section{Survival Outcomes}

Median follow-up was 74.8 months (range, 1.1-84.0 months). During the study period, 380 any-cause and 198 cause-specific deaths occurred. Overall, the SAHR models fitted the data well, with the Cox-Snell residuals following a unit-exponential distribution. In the unadjusted model, only women with the HR-/ HER2- cancer subtype had an increased hazard of dying 
Table 2. Study Cohort Characteristics and ORs of Receiving Guideline-Concordant AST

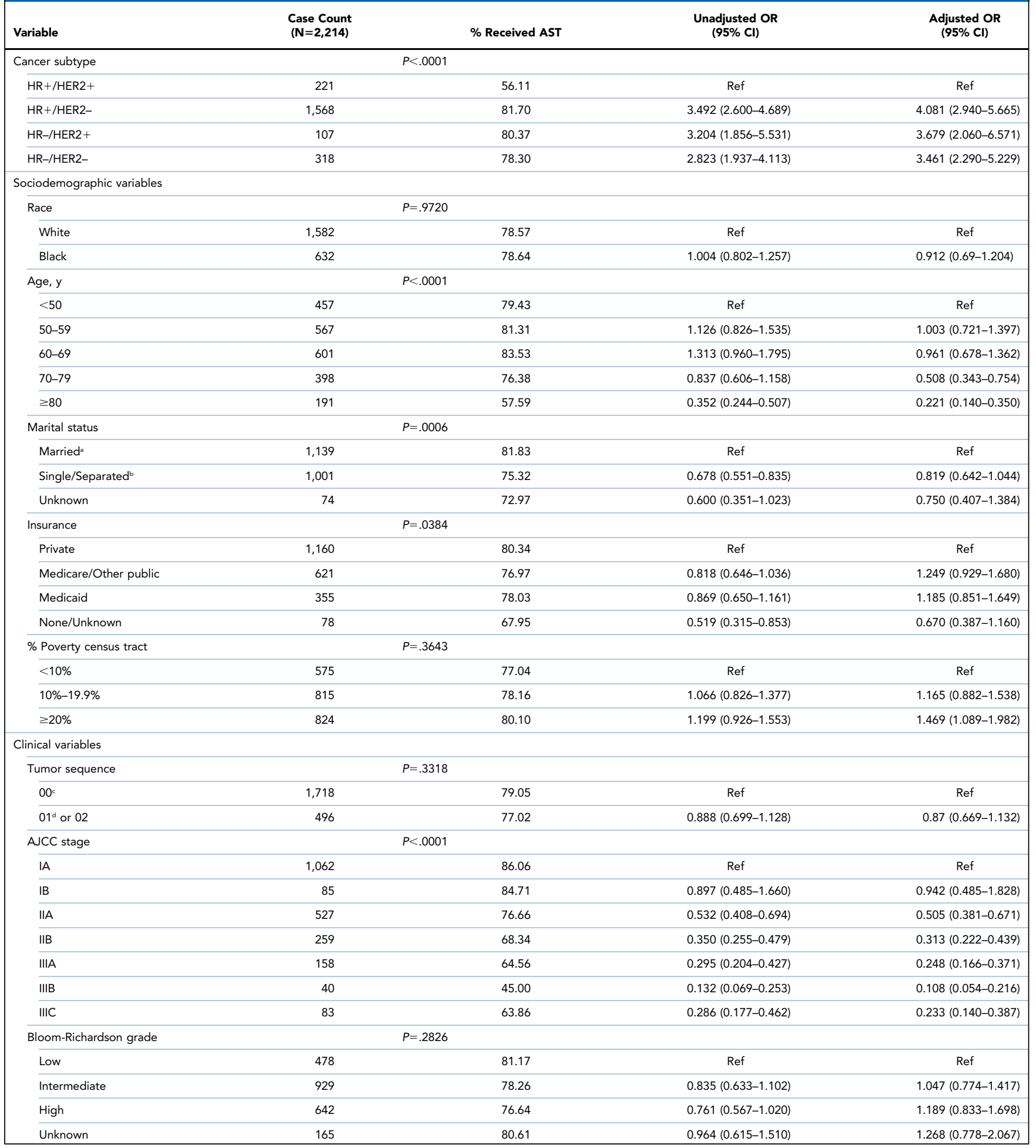

Variables included in the adjusted model were subtype, sociodemographic, and clinical.

Abbreviations: AST, adjuvant systemic therapy; CC, Charlson comorbidity; HR, hormone receptor; OR, odds ratio.

aMarried or living with partner.

bSingle, divorced, widowed, or separated.

cBreast cancer is the only primary cancer.

${ }^{d}$ Breast cancer is either the first or the second primary cancer. 


\begin{tabular}{|c|c|c|c|c|}
\hline Comorbidity & & & & \\
\hline Not documented & 862 & 77.38 & $0.993(0.794-1.242)$ & $0.930(0.728-1.187)$ \\
\hline No CC & 889 & 77.50 & Ref & Ref \\
\hline CC score $\geq 2$ & 95 & 77.89 & $1.023(0.615-1.703)$ & $1.483(0.841-2.615)$ \\
\hline
\end{tabular}

Variables included in the adjusted model were subtype, sociodemographic, and clinical.

Abbreviations: AST, adjuvant systemic therapy; CC, Charlson comorbidity; HR, hormone receptor; OR, odds ratio.

aMarried or living with partner.

bSingle, divorced, widowed, or separated.

'Breast cancer is the only primary cancer.

${ }^{d}$ Breast cancer is either the first or the second primary cancer.

of $0.0266(P=.0001)$ compared with those with the $\mathrm{HR}+$ I HER2 + subtype (Table 3), which implies that on average an additional 27 per 1,000 women with the HR-/HER2subtype died of any-cause death per year compared with those with the HR+/HER2+ subtype. However, for cause-specific survival, women with the HR-/HER2+ or the HR-/HER2- subtype had an increased hazard of dying of $0.0199(P=.0113)$ and $0.0283(P<.0001)$, respectively, compared with those with the HR+/HER2+ subtype. After adjusting for guideline-concordant AST and other factors, women with the HR-/HER2+ or the HR-/HER2- subtype had a significantly increased hazard of dying of any cause $(0.0216$ and 0.0266 , respectively) or causespecific death (0.0196 and 0.0262 , respectively) compared with those with the HR+/HER2+ subtype.

The survival probability for women who were guideline-discordant translated to a significantly increased hazard of dying of any cause and cause-specific death compared with those adhering to guidelines in both the unadjusted and the adjusted models (Table 3). However, the impact of treatment effect on survival reduced after adjustment with a hazard of 0.0322 to 0.0191 for overall survival and 0.0182 to 0.0126 for cause-specific survival. Other covariates, such as patients with Medicaid as the primary insurance, $>1$ tumor, and advanced cancer stage, showed an increased hazard of death for both overall and cause-specific survival outcomes, and an older age group, a

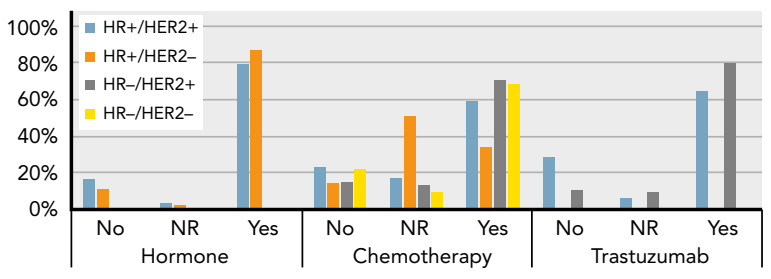

Figure 2. Percentage of patients with stage I-III breast cancer receiving adjuvant systemic therapy by treatment type and cancer subtype. Abbreviations: HR, hormone receptor; NR, treatment not required or was considered but not received. high Bloom-Richardson grade, and Charlson comorbidity were associated with risk of dying of any cause only when compared with their counterparts after adjustment.

When comparing survival among cancer subtypes stratified by guideline-concordant AST status (Figure 3), we found that among women who received recommended AST, those with the HR-/HER2- subtype had the worst survival in both survival outcomes (Figure 3A, C). Yet among guideline-discordant women, those with the HR-/HER2- subtype had worse survival than those with other subtypes observed before year 5 after cancer diagnosis for both any-cause and cause-specific death, whereas at the fifth year after cancer diagnosis, women with the HR-/HER2 + subtype had the worst survival (Figure 3B, D). Furthermore, we compared survival between patients who were guideline-concordant and guideline-discordant receiving AST stratified by cancer subtype (Figure 4). Overall, for both survival outcomes, those receiving AST had significantly better survival across all subtypes except cause-specific survival for patients with the HR+/HER2+ subtype, which was not a significant difference $(P=.2350$; Figure $4 \mathrm{E})$.

\section{Discussion}

Clinical decisions for AST for patients with breast cancer profoundly depend on their ER/PR and HER2 status. Although population-based cancer registries have been collecting ER and PR biomarkers from pathology reports and medical charts for $>2$ decades, it was not until the implementation of the second version of the Collaborative Stage Data Collection System, version $2{ }^{26}$ on January 1 , 2010, that the collection of HER2 test results was required. This is the first population-based study comprehensively evaluating the administration of complete NCCN-recommended AST by breast cancer subtype. In this study, breast cancer subtype was significantly associated with receiving guideline-concordant AST. Women with the HR+/HER2+ subtype had the lowest adherence rate $(56 \%)$ for recommended AST, which may be 
Table 3. Effect Estimate and SE From SAHR Model Fitting for Overall and Cause-Specific Survival

\begin{tabular}{|c|c|c|c|c|c|c|c|c|}
\hline \multirow[b]{2}{*}{ Variable } & \multicolumn{4}{|c|}{ Overall Survival } & \multicolumn{4}{|c|}{ Cause-Specific Survival } \\
\hline & $\begin{array}{l}\text { Unadjusted Model } \\
\text { Estimate (SE) }\end{array}$ & $P$ Value & $\begin{array}{l}\text { Adjusted Model } \\
\text { Estimate (SE) }\end{array}$ & $P$ Value & $\begin{array}{l}\text { Unadjusted Model } \\
\text { Estimate (SE) }\end{array}$ & $P$ Value & $\begin{array}{l}\text { Adjusted Model } \\
\text { Estimate (SE) }\end{array}$ & $P$ Value \\
\hline \multicolumn{9}{|l|}{ Cancer subtype } \\
\hline $\mathrm{HR}+/ \mathrm{HER} 2+$ & Ref & & Ref & & Ref & & Ref & \\
\hline $\mathrm{HR}+/ \mathrm{HER} 2-$ & $0.0031(0.0046)$ & .5029 & $0.0095(0.0050)$ & .0559 & $0.00004(0.0031)$ & .9945 & $0.0063(0.0035)$ & .0685 \\
\hline HR-/HER2+ & $0.0178(0.0095)$ & .0608 & $0.0216(0.0096)$ & .0248 & $0.0199(0.0079)$ & .0113 & $0.0196(0.0080)$ & .0146 \\
\hline HR-/HER2- & $0.0266(0.0069)$ & .0001 & $0.0266(0.0071)$ & .0002 & $0.0283(0.0056)$ & $<.0001$ & $0.0262(0.0058)$ & $<.0001$ \\
\hline \multicolumn{9}{|l|}{ Guideline-concordant } \\
\hline No & $0.0322(0.0050)$ & $<.0001$ & $0.0191(0.0050)$ & .0001 & $0.0182(0.0037)$ & $<.0001$ & $0.0126(0.0039)$ & .0011 \\
\hline Yes & Ref & & Ref & & Ref & & Ref & \\
\hline \multicolumn{9}{|l|}{ Sociodemographic } \\
\hline \multicolumn{9}{|l|}{ Race } \\
\hline White & Ref & & Ref & & Ref & & Ref & \\
\hline Black & $0.0088(0.0036)$ & .0157 & $0.0006(0.0040)$ & .8747 & $0.0135(0.0029)$ & $<.0001$ & $0.0060(0.0030)$ & .0484 \\
\hline \multicolumn{9}{|l|}{ Age, y } \\
\hline$<50$ & Ref & & Ref & & Ref & & Ref & \\
\hline $50-59$ & $-0.0059(0.0037)$ & .1127 & $-0.0018(0.0038)$ & .6291 & $-0.0061(0.0034)$ & .0741 & $-0.0014(0.0034)$ & .6758 \\
\hline $60-69$ & $-0.0017(0.0038)$ & .6540 & $0.0049(0.0042)$ & .2397 & $-0.0083(0.0032)$ & .0110 & $-0.0005(0.0035)$ & .8944 \\
\hline $70-79$ & $0.0191(0.0052)$ & .0003 & $0.0228(0.0061)$ & .0002 & $-0.0040(0.0038)$ & .2975 & $0.0021(0.0045)$ & .6477 \\
\hline$\geq 80$ & $0.0895(0.0117)$ & $<.0001$ & $0.0863(0.0124)$ & $<.0001$ & $0.0102(0.0065)$ & .1148 & $0.0131(0.0072)$ & .0701 \\
\hline \multicolumn{9}{|l|}{ Marital status } \\
\hline Married ${ }^{\mathrm{a}}$ & Ref & & Ref & & Ref & & Ref & \\
\hline Single/Separated ${ }^{\mathrm{b}}$ & $0.0222(0.0033)$ & $<.0001$ & $0.0047(0.0033)$ & .1570 & $0.0095(0.0024)$ & .0001 & $0.0016(0.0024)$ & .4999 \\
\hline Unknown & $-0.0021(0.0069)$ & .7548 & $-0.0101(0.0071)$ & .1569 & $0.00003(0.0054)$ & .996 & $-0.0046(0.0057)$ & .4229 \\
\hline \multicolumn{9}{|l|}{ Insurance } \\
\hline Private & Ref & & Ref & & Ref & & Ref & \\
\hline Medicare/Other public & $0.0212(0.0039)$ & $<.0001$ & $-0.0004(0.0046)$ & .9333 & $0.0048(0.0026)$ & .0601 & $0.0023(0.0030)$ & .4430 \\
\hline Medicaid & $0.0289(0.0053)$ & $<.0001$ & $0.0178(0.0055)$ & .0012 & $0.0178(0.0041)$ & $<.0001$ & $0.0096(0.0042)$ & .0222 \\
\hline None/Unknown & $0.0005(0.0070)$ & .9435 & $-0.0026(0.0072)$ & .7169 & $-0.0003(0.0052)$ & .9471 & $-0.0049(0.0055)$ & .3695 \\
\hline \multicolumn{9}{|l|}{$\%$ Poverty census tract } \\
\hline$<10 \%$ & Ref & & Ref & & Ref & & Ref & \\
\hline $10 \%-19.9 \%$ & $0.0050(0.0029)$ & .0828 & $-0.0007(0.0037)$ & .8621 & $0.0047(0.0026)$ & .0673 & $0.0023(0.0026)$ & .3840 \\
\hline$\geq 20 \%$ & $0.0096(0.0031)$ & .0018 & $0.0004(0.0041)$ & .9157 & $0.0085(0.0027)$ & .0018 & $0.0019(0.0029)$ & .5134 \\
\hline \multicolumn{9}{|l|}{ Tumor sequence } \\
\hline $00^{c}$ & Ref & & Ref & & Ref & & Ref & \\
\hline $01^{\mathrm{d}}$ or 02 & $0.0248(0.0046)$ & $<.0001$ & $0.0228(0.0046)$ & $<.0001$ & $0.0052(0.003)$ & .0808 & $0.0075(0.0031)$ & .0155 \\
\hline \multicolumn{9}{|l|}{ AJCC stage } \\
\hline 1 & Ref & & Ref & & Ref & & Ref & \\
\hline ॥ & $0.0157(0.0032)$ & $<.0001$ & $0.0136(0.0033)$ & $<.0001$ & $0.0121(0.0022)$ & $<.0001$ & $0.0079(0.0022)$ & .0004 \\
\hline III & $0.0632(0.0079)$ & $<.0001$ & $0.0581(0.0080)$ & $<.0001$ & $0.0535(0.0067)$ & $<.0001$ & $0.0472(0.0067)$ & $<.0001$ \\
\hline \multicolumn{9}{|l|}{ Bloom-Richardson grade } \\
\hline Low & Ref & & Ref & & Ref & & Ref & \\
\hline Intermediate & $0.0046(0.0036)$ & .2029 & $0.0006(0.0037)$ & .8736 & $0.0020(0.0023)$ & .3840 & $-0.0031(0.0023)$ & .1901 \\
\hline High & $0.0211(0.0045)$ & $<.0001$ & $0.0130(0.0052)$ & .0126 & $0.0212(0.0034)$ & $<.0001$ & $0.0074(0.0038)$ & .0544 \\
\hline Unknown & $-0.0030(0.0053)$ & .5750 & $-0.0075(0.0056)$ & .1769 & $0.0026(0.0039)$ & .5041 & $-0.0068(0.0040)$ & .0946 \\
\hline
\end{tabular}

Variables included in the adjusted model were subtype, guideline-concordant adjuvant systemic therapy, sociodemographic, and clinical.

(continued on next page)

Abbreviations: CC, Charlson comorbidity; HR, hormone receptor; SAHR, semiparametric additive hazard regression model; SE, standard error

a Married or living with partner.

bSingle, divorced, widowed, or separated.

cBreast cancer is the only primary cancer.

${ }^{\mathrm{d} B r e a s t}$ cancer is either the first or the second primary cancer. 
Table 3. Effect Estimate and SE From SAHR Model Fitting for Overall and Cause-Specific Survival (cont.)

\begin{tabular}{|c|c|c|c|c|c|c|c|c|}
\hline \multirow[b]{2}{*}{ Variable } & \multicolumn{4}{|c|}{ Overall Survival } & \multicolumn{4}{|c|}{ Cause-Specific Survival } \\
\hline & $\begin{array}{l}\text { Unadjusted Model } \\
\text { Estimate (SE) }\end{array}$ & $P$ Value & $\begin{array}{l}\text { Adjusted Model } \\
\text { Estimate (SE) }\end{array}$ & $P$ Value & $\begin{array}{l}\text { Unadjusted Model } \\
\text { Estimate (SE) }\end{array}$ & $P$ Value & $\begin{array}{l}\text { Adjusted Model } \\
\text { Estimate (SE) }\end{array}$ & $P$ Value \\
\hline \multicolumn{9}{|l|}{ Sociodemographic (cont.) } \\
\hline \multicolumn{9}{|l|}{ Comorbidity } \\
\hline Not documented & $-0.0037(0.0031)$ & .2358 & $-0.0002(0.0032)$ & .9576 & $-0.0027(0.0024)$ & .2593 & $-0.0014(0.0025)$ & .5812 \\
\hline No CC & Ref & & Ref & & Ref & & Ref & \\
\hline CC score 1 & $0.0174(0.0052)$ & .0009 & $0.0117(0.0053)$ & .0269 & $0.0027(0.0035)$ & .4338 & $0.0016(0.0035)$ & .6466 \\
\hline$C C$ score $\geq 2$ & $0.0564(0.0135)$ & $<.0001$ & $0.0437(0.0134)$ & .0011 & $0.0161(0.0084)$ & .0559 & $0.0117(0.0084)$ & .1635 \\
\hline
\end{tabular}

Variables included in the adjusted model were subtype, guideline-concordant adjuvant systemic therapy, sociodemographic, and clinical.

Abbreviations: CC, Charlson comorbidity; HR, hormone receptor; SAHR, semiparametric additive hazard regression model; SE, standard error.

aMarried or living with partner.

bSingle, divorced, widowed, or separated.

'Breast cancer is the only primary cancer.

dBreast cancer is either the first or the second primary cancer.

attributed to all 3 regimens of systemic therapy (hormone, chemotherapy, trastuzumab) being recommended for early-stage disease (stage I, II, and IIIA with N1), except for stage I disease with tumor size $\leq 1 \mathrm{~cm}$ or favorable histologies (tubular and colloid). Compliance rates for other subtypes were similar, at approximately $80 \%$.

Among sociodemographic variables, age was inversely associated with receipt of AST and remained highly significant after adjusting for cancer subtype and other variables, consistent with previous studies. ${ }^{12,13,27}$ We observed no differences in guideline concordance for women aged $<70$ years, whereas adherence to AST decreased significantly for those aged $\geq 70$ years. In addition, women living in a census tract $\geq 20 \%$ below poverty had a higher adherence rate for AST, which could be attributed to Louisiana's unique public hospital system that provides free standard care to patients who are underserved. Regarding the association between receipt of AST and clinical variables, our results indicate that the likelihood of guideline concordance decreases with advanced disease stages and that women with mild comorbidity (CCS 1) are more likely to be guideline-concordant than those without comorbidity, which contradicts findings of other studies. ${ }^{12}$ One possible explanation is that previous studies excluded cases for which adjuvant therapy was "considered," whereas our study categorized "considered" systemic therapy as guidelineconcordant despite whether the treatment was received.

Numerous clinical trials have demonstrated the benefit of specific systemic therapy for HR+ or HER2+ breast cancer. By jointly considering all AST, we found that women who were guideline-concordant significantly improved in both survival outcomes across the 4 subtypes. The study revealed that women with HR+ disease regardless of HER2 status had almost identical cause-specific survival among those receiving guidelinerecommended AST. Most studies have found that patients with the HR+/HER2- subtype had the best survival rate among subtypes ${ }^{28-31}$; nevertheless, our data showed that women with HR+/HER2+ tumors had the best survival outcomes except for cause-specific survival in the guideline-concordant cohort. Another key finding from this study is that among patients with HR-, those with HER2+ had better overall and cause-specific survival than those with HER2- when they received NCCN-recommended chemotherapy and trastuzumab. Survival differences in these patients were amplified with longer observation. This result confirmed the effect of trastuzumab after chemotherapy for HER2+ tumors, demonstrated in several clinical trials, ${ }^{8,9}$ particularly in HR- cohorts. Although $80 \%$ of patients with HR-/HER2were guideline-concordant, their survival rate was worst among the 4 subtypes in both overall and cause-specific death, primarily because there are no effective biomarkertargeted therapies to prolong patient survival and maintain a high relapse rate, ${ }^{32,33}$ and the only option for treatment in these patients is conventional chemotherapy.

We found that women aged $\geq 70$ years were less likely to be guideline-concordant and had an increased hazard in overall survival, but not in cause-specific survival, compared with their counterparts. After stratifying by age group, we found that women aged $\geq 70$ years who were guidelineconcordant had a significantly improved overall survival $(P=.0009)$. Treating elderly patients with breast cancer is a complex process, and several factors such as life expectancy, existing comorbidity, treatment complications, benefit, and quality of life are usually considered in treatment decision-making. However, elderly patients with breast cancer should be informed of the standard adjuvant treatment and available options that would benefit them. ${ }^{34}$

This study has several strengths. First, it is a population-based study, using high-quality data from a statewide cancer registry. Second, complete treatment information was collected from all sources, including 
A

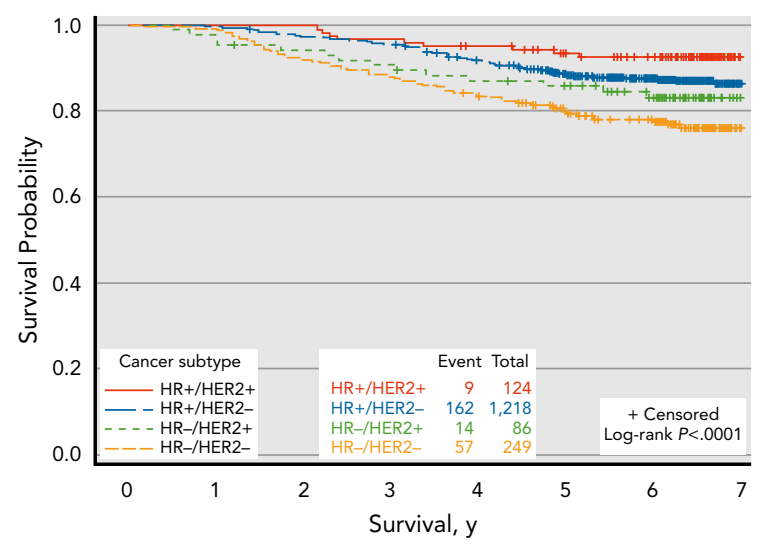

C

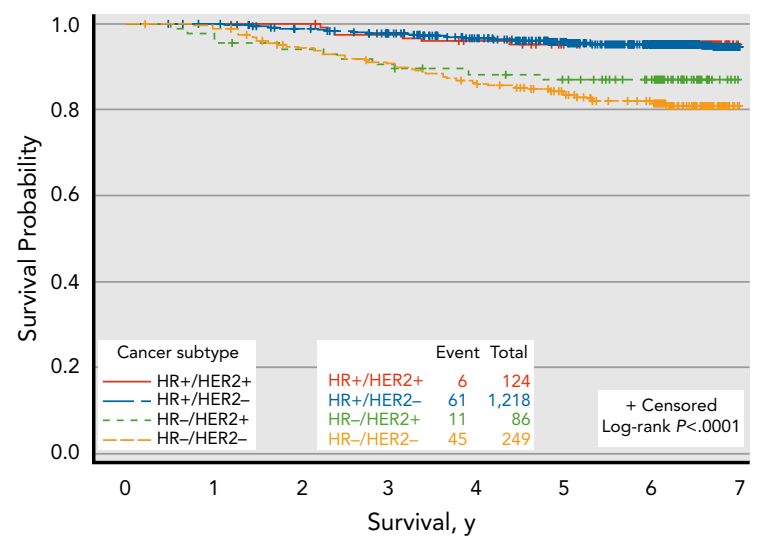

B

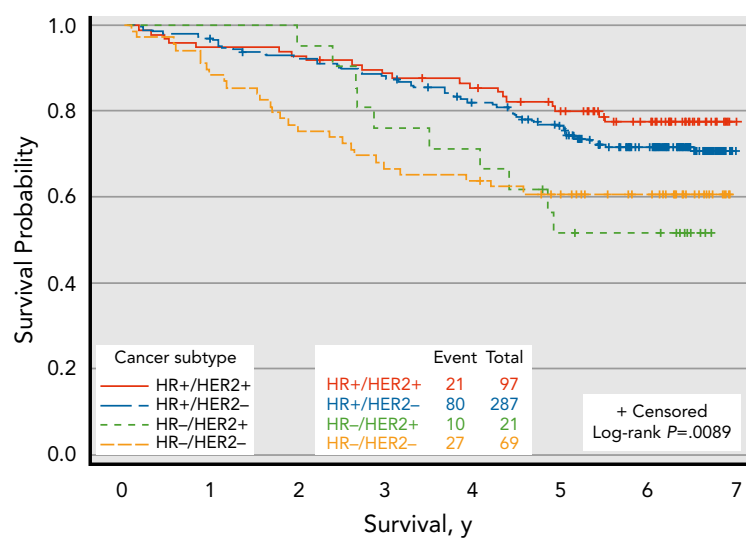

D

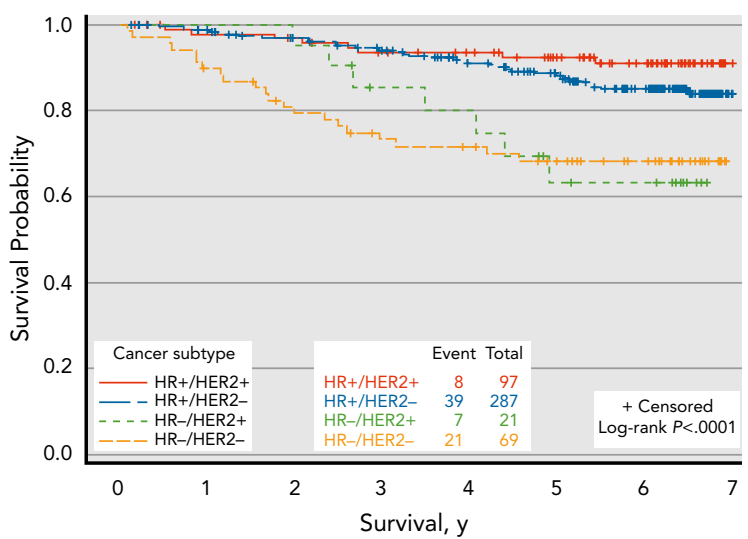

Figure 3. Kaplan-Meier survival curves by subtype for (A) overall survival for guideline-concordant AST, (B) overall survival for guideline-discordant AST, (C) cause-specific survival for guideline-concordant AST, and (D) cause-specific survival for guideline-discordant AST.

Abbreviations: AST, adjuvant systemic therapy; HR, hormone receptor.

physicians' offices, for all types of systemic therapy, including the Cancer Chemotherapy National Service Center (NSC) number on systemic therapy drugs and the completion status of chemotherapy. This comprehensive process enabled us to separate trastuzumab (NSC number: 688097), which is specifically recommended for patients with HER2+, from chemotherapy drugs due to the classification. Third, the linkage of LTR data with the Oncotype DX database allowed us to capture recurrence risk score from multigene signature tests and supplement this site-specific factor in the registry data. The recurrence risk score is an important determinant in chemotherapy administration for patients with node-negative, HR+/HER2-, early-stage breast cancer per the NCCN Guidelines. ${ }^{11}$

A few limitations should be noted. First, information was lacking on the rationale behind a physician's decision whether to give AST to patients with disease for which the NCCN Guidelines recommend adjuvant therapy be "considered" with certain systemic therapy. In addition, administering recommended adjuvant chemotherapy to patients aged $\geq 70$ years may vary by physician based on the approach to this age group when taking into consideration patients' existing comorbid conditions, life expectancy, or preference. Another limitation was using the immunohistochemical-based (and/or FISH-based) surrogate cancer subtype to estimate survival, which may not be comparable to gene-expression profile cancer subtype. However, this study demonstrated that the benefits of adhering to guideline-concordant AST among subtypes are the same as in clinical trials based on gene-expression profiling. Finally, the follow-up time may not have been long enough to capture adequate events for subtypes that had a longer survival time, such as $\mathrm{HR}+$, to ensure estimated statistical tests with sufficient power, ${ }^{35}$ particularly in cause-specific survival.

\section{Conclusions}

This study showed that in a community setting, women with breast cancer had better survival outcomes if they 
A

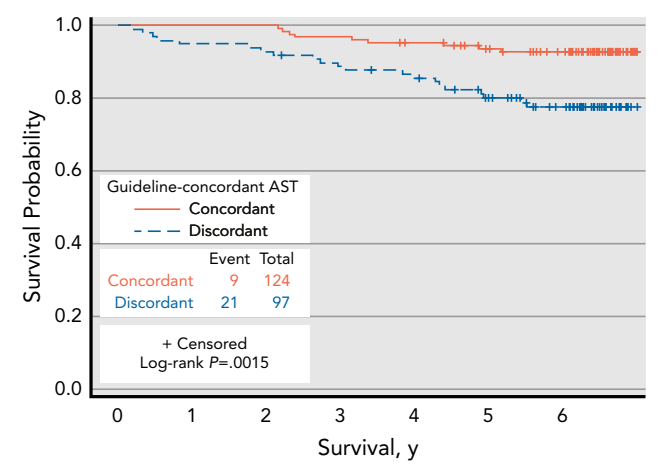

C

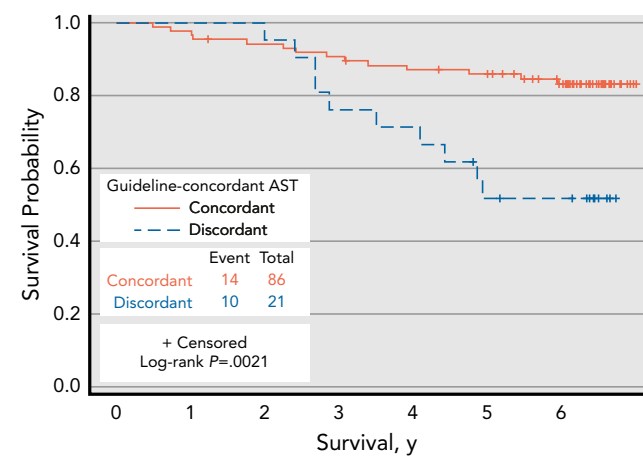

E

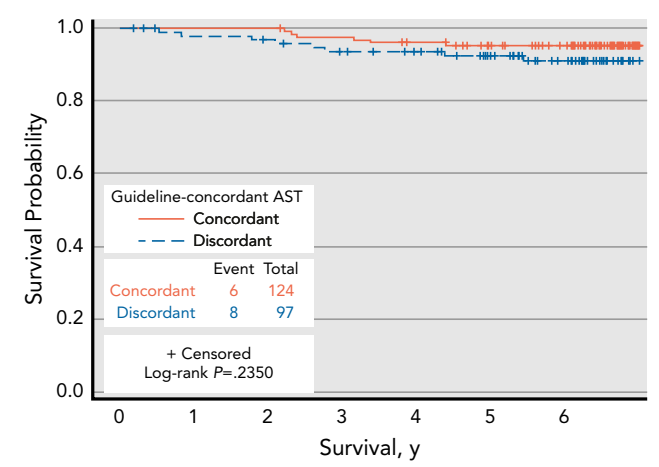

G

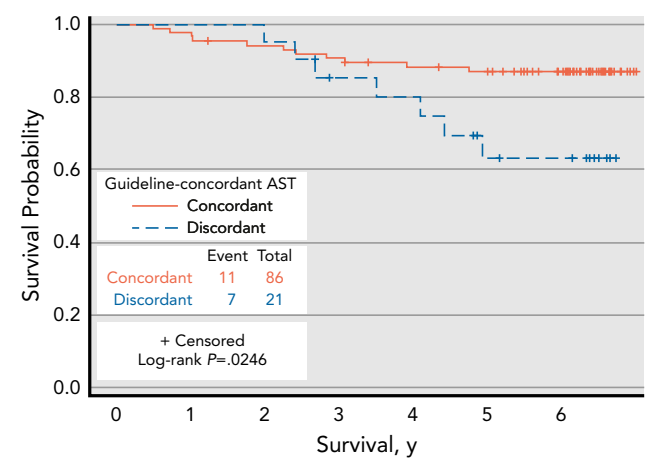

B

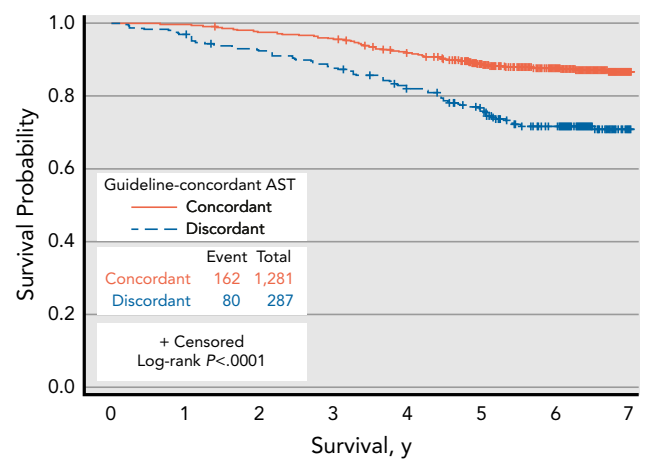

D

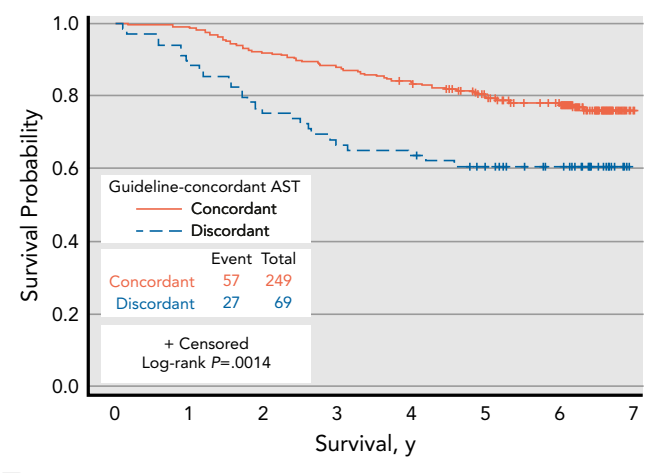

$F$

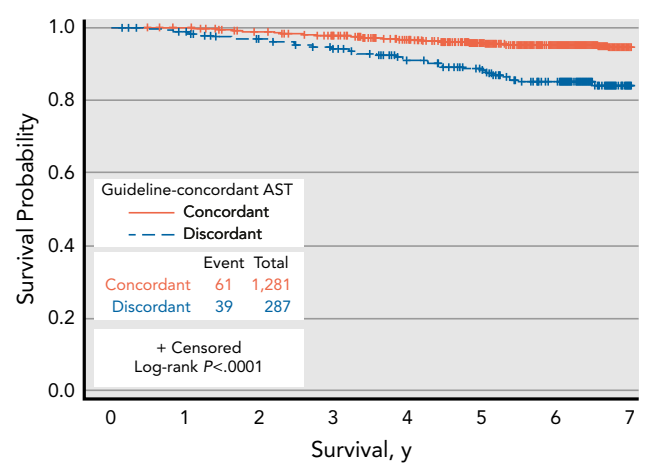

H

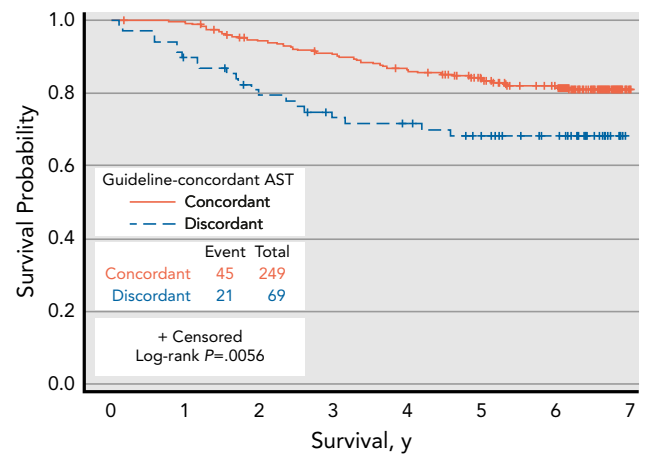

Figure 4. Kaplan-Meier survival curves by guideline-concordant AST status stratified by cancer subtype among women with stage I-III breast cancer. Overall survival for (A) HR+/HER2+, (B) HR+/HER2-, (C) HR-/HER2+, and (D) HR-/HER2-. Cause-specific survival for (E) HR+/HER2+, (F) HR+/HER2-, (G) HR-/HER2+, and (H) HR-/HER2-.

Abbreviations: AST, adjuvant systemic therapy; HR, hormone receptor. 
adhered to guideline-concordant AST. Although women with the HR+/HER2+ subtype had the lowest rate of receiving guideline-concordant treatment, their overall and cause-specific survival rates were the best among the 4 subtypes. In contrast, patients with HR-/HER2- had the worst survival outcomes even with a high rate of guidelineadherent treatment. Advances in treatment options other than chemotherapy are urgently needed to improve survival for women with HR-/HER2- breast cancer.

Submitted September 4, 2018; accepted for publication January 9, 2019.

Author contributions: Study concept: Hsieh, Chen. Study design: Hsieh, Wu Data collection and quality assessment: Davidson. Data analysis: Hsieh, Zhang.
Guidance on clinical practice of breast cancer treatment: Loch Writing —original draft and revision: Hsieh. Writing—review and editing: Zhang, Wu, Davidson, Loch, Chen.

Disclosures: The authors have not received any financial consideration from any person or organization to support the preparation, analysis, results, or discussion of this article.

Funding: This work was supported in part by the CDC Cooperative Agreements of the National Program of Cancer Registries (grant/award number: U58DP003915) in conjunction with a CDC Comparative Effectiveness Research contract to ICF (grant/award number: 635243-10S-1566) and NCl's contract number HHSN2612018000071.

Disclaimer: The findings and conclusions are those of the authors and do not necessarily represent the official position of their affiliations, the $\mathrm{CDC}$, or $\mathrm{NCl}$.

Correspondence: Mei-Chin Hsieh, PhD, Epidemiology Program and Louisiana Tumor Registry, School of Public Health, Louisiana State University Health Sciences Center, 2020 Gravier Street, New Orleans, LA 70112. Email: mhsieh@|suhsc.edu

\section{References}

1. Perou CM, Sørlie T, Eisen MB et al. Molecular portraits of human breast tumours. Nature 2000;406:747-752.

2. Sørlie T, Perou CM, Tibshirani R, et al. Gene expression patterns of breast carcinomas distinguish tumor subclasses with clinical implications. Proc Natl Acad Sci USA 2001;98:10869-10874.

3. Sotiriou C, Neo SY, McShane LM, et al. Breast cancer classification and prognosis based on gene expression profiles from a population-based study. Proc Natl Acad Sci USA 2003;100:10393-10398.

4. Sotiriou C, Pusztai L. Gene-expression signatures in breast cancer. N Engl J Med 2009;360:790-800.

5. Carey LA, Perou CM, Livasy CA, et al. Race, breast cancer subtypes, and survival in the Carolina Breast Cancer Study. JAMA 2006;295:2492-2502.

6. Early Breast Cancer Trialists' Collaborative Group. Effects of chemotherapy and hormonal therapy for early breast cancer on recurrence and 15-year survival: an overview of the randomised trials. Lancet 2005;365: 1687-1717.

7. Berry DA, Cirrincione C, Henderson IC, et al. Estrogen-receptor status and outcomes of modern chemotherapy for patients with node-positive breast cancer. JAMA 2006;295:1658-1667.

8. Piccart-Gebhart MJ, Procter M, Leyland-Jones B, et al. Trastuzumab after adjuvant chemotherapy in HER2-positive breast cancer. N Engl J Med 2005;353:1659-1672.

9. Slamon D, Eiermann W, Robert N, et al. Adjuvant trastuzumab in HER2 positive breast cancer. N Engl J Med 2011;365:1273-1283.

10. Harris L, Fritsche H, Mennel R, et al. American Society of Clinical Oncology 2007 update of recommendations for the use of tumor markers in breast cancer. J Clin Oncol 2007;25:5287-5312.

11. Gradishar WJ, Anderson BO, Abraham J, et al. NCCN Clinical Practice Guidelines in Oncology: Breast Cancer. Version 1.2019. Accessed March 15,2019 . To view the most recent version, visit NCCN.org

12. Wu XC, Lund MJ, Kimmick GG, et al. Influence of race, insurance, socioeconomic status, and hospital type on receipt of guideline-concordant adjuvant systemic therapy for locoregional breast cancers. J Clin Oncol 2012;30:142-150.

13. Guy GP Jr, Lipscomb J, Gillespie TW, et al. Variations in guidelineconcordant breast cancer adjuvant therapy in rural Georgia. Health Serv Res 2015;50:1088-1108.

14. Zurawska U, Baribeau DA, Giilck S, et al. Outcomes of HER2-positive early-stage breast cancer in the trastuzumab era: a population-based study of Canadian patients. Curr Oncol 2013;20:e539-545.

15. Chen VW, Eheman CR, Johnson CJ, et al. Enhancing cancer registry data for comparative effectiveness research (CER) project: overview and methodology. J Registry Manag 2014;41:103-112.

16. Howlader $\mathrm{N}$, Altekruse SF, Li Cl, et al. US incidence of breast cancer subtypes defined by joint hormone receptor and HER2 status. J Nat Cancer Inst 2014;106:pii:dju055.

17. Paik S. Development and clinical utility of a 21-gene recurrence score prognostic assay in patients with early breast cancer treated with tamoxifen. Oncologist 2007;12:631-635.
18. Sparano JA, Paik S. Development of the 21-gene assay and its application in clinical practice and clinical trials. J Clin Oncol 2008 26:721-728

19. Quan H, Sundararajan V, Halfon $P$, et al. Coding algorithms for defining comorbidities in ICD-9-CM and ICD-10 administrative data. Med Care 2005;43:1130-1139.

20. National Cancer Institute. SEER cause-specific death classification Available at: http://seer.cancer.gov/causespecific/index.html. Accessed September 10, 2017.

21. American College of Surgeons, Commission on Cancer. Facility Oncology Registry Data Standards. Revised for 2011. Available at: https://www.facs. org/ /media/files/quality\%20programs/cancer/coc/fords/fords_for_ 2011_01012011.ashx. Accessed May 25, 2017.

22. Lin DY, Ying Z. Semiparametric analysis of the additive risk model. Biometrika 1994;81:61-71

23. Schaubel DE, Wei G. Fitting semiparametric additive hazards models using standard statistical software. Biom J 2007;49:719-730.

24. Klein JP, Moeschberger ML. Survival Analysis: Techniques for Censored and Truncated Data, 2nd edition. New York, NY: Springer-Verlag; 1997.

25. Xie X, Strickler HD, Xue X. Additive hazard regression models: an application to the natural history of human papillomavirus. Comput Math Methods Med 2013;796270. http://dx.doi.org/10.1155/2013/ 796270 .

26. Collaborative Stage Data Collection System, Version 02.05. https:// cancerstaging.org/cstage/schema/Pages/version0205.aspx. Accessed May 25, 2017

27. Verschoor AM, Kuijer A, Verloop J, et al. Adjuvant systemic therapy in early breast cancer: impact of guideline changes and clinicopathological factors associated with nonadherence at a nation-wide level. Breast Cancer Res Treat 2016;159:357-365.

28. O'Brien KM, Cole SR, Tse CK, et al. Intrinsic breast tumor subtypes, race, and long-term survival in the Carolina Breast Cancer Study. Clin Cancer Res 2010;16:6100-6110.

29. Parise CA, Caggiano V. Breast cancer survival defined by the ER/PR/HER2 subtypes and a surrogate classification according to tumor grade and immunohistochemical biomarkers. J Cancer Epidemiol 2014;2014:469251.

30. Fallahpour S, Navaneelan T, De P, et al. Breast cancer survival by molecular subtype: a population-based analysis of cancer registry data. CMAJ Open 2017;5:E734-739.

31. Haque R, Ahmed SA, Inzhakova G, et al. Impact of breast cancer subtypes and treatment on survival: an analysis spanning two decades. Cancer Epidemiol Biomarkers Prev 2012;21:1848-1855.

32. Haffty BG, Yang $Q$, Reiss $M$, et al. Locoregional relapse and distant metastasis in conservatively managed triple negative early-stage breast cancer. J Clin Oncol 2006;24:5652-5657.

33. Shim HJ, Kim SH, Kang BJ, et al. Breast cancer recurrence according to molecular subtype. Asian Pac J Cancer Prev 2014;15:5539-5544.

34. Leone J, Leone BA, Leone JP. Adjuvant systemic therapy in older women with breast cancer. Breast Cancer (Dove Med Press) 2016;8:141-147

35. Clark TG, Bradburn MJ, Love SB, et al. Survival analysis part I: basic concepts and first analyses. Br J Cancer 2003;89:232-238. 